\title{
Glycoconjugate Expression in Normal, Metaplastic, and Neoplastic Human Upper Gastrointestinal Mucosa
}

\author{
Chikao Shimamoto, ${ }^{*}$ Wilfred M. Weinstein, ${ }^{\ddagger}$ and C. Richard Boland* \\ *Department of Medicine and the Michigan Gastrointestinal Peptide Research Center, University of Michigan School of Medicine, \\ Ann Arbor, Michigan 48105; Ann Arbor Veterans Administration Medical Center, Ann Arbor, Michigan 48105; and ${ }^{\ddagger}$ Department of \\ Medicine and Center for Ulcer Research and Education (CURE), University of California, Los Angeles School of Medicine, \\ Center for the Health Sciences, Los Angeles, California 90024
}

\begin{abstract}
Glycoconjugate structure in upper gastrointestinal epithelium was studied using five lectins to determine the relationship between aberrant differentiation and glycoconjugate expression. Specimens of normal esophagus, stomach, and duodenum were examined and compared with specimens of columnar metaplasia in the esophagus (Barrett's esophagus) and specimens of adenocarcinoma of the esophagus and stomach. Specific terminal glycoconjugate structures were found for the esophagus, stomach, and duodenum. Minor differences were found between the antral and fundic gland mucosae, reflecting their respective cell populations. In biopsies of Barrett's esophagus, gastric-type columnar metaplasia expressed glycoconjugates indistinguishable from those in the normal stomach. In specialized-type columnar metaplasia, a more restricted expression of glycoconjugates was seen resembling the normal duodenum. The presence of low grade dysplasia in Barrett's esophagus associated with adenocarcinoma had no impact on glycoconjugate expression. However, a distinctive difference in glycosylation was seen in high grade dysplasia of the columnar-lined esophagus and in adenocarcinoma of the esophagus and stomach. Barrett's esophagus is a morphological mosaic in which the glycoconjugate expression resembles that seen in the normal stomach and duodenum. However, in high grade dysplasia and carcinoma, variable deletion of glycoconjugate expression can be found.
\end{abstract}

\section{Introduction}

Glycoconjugates are abundantly expressed in gastrointestinal epithelium, and play a central role in a wide range of cellular behavior (1). Much of the heterogeneity expressed among glycoconjugates is due to structural variations in the carbohydrate portion of the molecule (2). Specifically, although only five different monosaccharide residues may be found in mammalian gastrointestinal mucins, ${ }^{1}$ an extremely rich range of diver-

Address correspondence to Dr. Boland, Gastrointestinal Unit (111D), Veterans Administration Medical Center, 2215 Fuller Road, Ann Arbor, MI 48105.

Received for publication 24 September 1986 and in revised form 10 August 1987.

1. The term mucus refers to the viscid secretory product of mucus-secreting epithelium. Mucous is the adjective derived from the noun mucus. Mucin is the high molecular weight glycoprotein that imparts the physical characteristics to, and excepting water is the principal

The Journal of Clinical Investigation, Inc. Volume 80, December 1987, 1670-1678 sity may be generated by differences in the carbohydrate sequences and anomeric linkages between adjacent sugar residues $(3,4)$.

Certain antigenic structures may be recognized in glycoproteins that are associated with the process of normal cellular differentiation $(5,6)$. The mechanism for this is in part due to differential expression of glycosyltransferase activities during the process of differentiation (6). Different forms of mucin are expressed not only as a function of location within the gastrointestinal tract $(7,8)$, but also in association with the process of differentiation (9). In addition, a specific form of mucin not seen in normal colon is expressed in benign and malignant colonic neoplasms, which may be detected using lectin histochemical techniques $(9,10)$. Gastrointestinal mucins have attracted interest because their structural diversity reflects modifications in glycoprotein synthesis and processing that accompany cellular differentiation, neoplasia, inflammatory bowel disease (11), and possibly peptic ulcer disease (12).

A paradigm of aberrant cellular differentiation occurring in response to chronic injury is the phenomenon of columnar metaplasia in the esophagus in the setting of chronic gastroesophageal reflux (Barrett's esophagus). In this disease process, squamous epithelium is progressively replaced by columnar epithelium in a cephalad direction, and an increased risk for the development of adenocarcinoma is observed (13). This epithelium has been characterized histologically (14), in terms of cell kinetics (15), and histochemically (16-18). However, traditional histochemical techniques are limited in their ability to define the structure of mucins.

We have previously utilized fluorescent lectins to characterize differences in structure that occur with differentiation and in a variety of neoplastic and preneoplastic lesions in the colon $(19,20)$. Lectins are available that will identify and localize a variety of terminal carbohydrate structures on fixed sections of tissue. Thus, it was hypothesized that this same technique would permit the identification of differentiation-associated structures in mucins of the upper gastrointestinal tract. Therefore, a study was designed in which mucosal biopsies were obtained from patients with Barrett's esophagus and compared with sections obtained from the esophagus, stomach, and duodenum of these patients. Specimens of gastric adenocarcinoma, esophageal adenocarcinoma, and dysplasia arising in the setting of Barrett's epithelium were also investigated for comparative purposes.

component of, mucus. A mucin-type glycoprotein is a biochemical term for a glycoprotein containing oligosaccharide chains that are covalently linked through an $O$-glycosidic bond (therefore termed " $O$ linked") between the reducing carbon of $n$-acetylgalactosamine and the amino acids threonine or serine. 


\section{Methods}

Tissues. Biopsies from 10 patients with Barrett's esophagus without carcinoma or high grade dysplasia were obtained at endoscopy. In each patient, biopsies were obtained from the esophagus, stomach, and duodenal bulb. In the esophagus, biopsies were taken beginning at the upper margin of the lower esophageal sphincter region and at $2-\mathrm{cm}$ intervals proximally. Each patient had one biopsy containing squamous epithelium taken proximal to the Barrett's zone. In the Barrett's zone, 49 esophageal biopsies were obtained from the 10 patients, $5 \pm 1$ biopsies per patient spanning $5.8 \pm 1.2 \mathrm{~cm}$ in the distal esophagus. The gastric and duodenal biopsies were taken as follows: duodenal bulb, distal antrum ( $1 \mathrm{~cm}$ proximal to the pylorus), and the proximal stomach ( $2 \mathrm{~cm}$ below the lower esophageal sphincter region).

All biopsies were taken with the jumbo pinch biopsy forceps (Olympus FB 13K). Each sample was oriented mucosal-side-up on monofilament plastic mesh (21) and was fixed in modified Bouin's solution containing $1 \%$ acetic acid. $4-\mu \mathrm{m}$ serial sections were prepared for conventional light microscopy. Approximately half the sections were stained with hematoxylin and eosin and the remainder were stained with hematoxylin and eosin-Alcian Blue, $\mathrm{pH} 2.5$.

All the histology was graded independently by Dr. Weinstein without prior knowledge of the lectin-staining results. The biopsies containing columnar epithelium in the esophagus were assessed with separate notations for the type of surface epithelium and for the underlying gland types, similar to that described previously (22). The surface epithelium was noted as either gastric or specialized type. Glands beneath the lining epithelium were characterized separately as fundic, cardiacfundic, or mucous type. A gastric type of surface epithelium in the esophagus referred to the appearance of gastric-type mucous-lining cells that stained negatively (i.e., colorless) with the hematoxylin and eosin-Alcian Blue, pH 2.5 stain. Specialized epithelium referred to surface epithelium that contained blue-staining goblet cells or cells that looked gastric in type but stained blue with the Alcian Blue stain. If most but not all of the surface epithelium was of one type, it was designated on the basis of the predominant type (gastric or specialized). 3 of the 49 biopsies that were designated specialized actually contained substantial areas of gastric-type lining epithelium. Therefore, for the lectin-staining studies these biopsies were analyzed separately for both their gastric-type and specialized-type of staining pattern. Overall, 27 biopsies or portions of biopsies were designated as gastric-type and 25 as specialized for the lectin-binding studies.

The majority of the biopsies from Barrett's esophagus contained only mucous-type glands beneath the lining epithelium. However, in 9 of the 49 biopsies, the underlying glands were of fundic gland or cardiac-fundic (mixed) gland type. These biopsies were all from the lower esophageal sphincter region.

The 10 duodenal biopsies were normal, as were five of the ten antral biopsies. The other five antral biopsies exhibited superficial or full-thickness mucosal gastritis. All 10 biopsies from proximal stomach contained fundic gland mucosa; there were mixed fundic-cardiac glands in two. Two of the ten fundic gland biopsies had inflammatory changes, superficial in one and focal atrophic in the other. Three gastric biopsies (two antral gland, 1 fundic gland) contained areas of intestinal metaplasia. One duodenal biopsy contained gastric surface cell metaplasia.

Specimens of neoplasia were obtained as follows. Two surgical resections and two endoscopic biopsy specimens of esophageal adenocarcinoma were obtained and fixed in buffered formalin. One of these cancers occurred in the documented setting of Barrett's esophagus, but columnar metaplasia could not be documented in the other three instances. All of the specimens of esophageal cancer were histologically well or moderately differentiated adenocarcinoma. Seven endoscopic biopsies of esophageal adenocarcinoma occurring in the setting of Barrett's esophagus were obtained and fixed in Bouin's solution. Each of the specimens of esophageal and gastric adenocarcinoma came from different patients.

Ten surgical specimens and two endoscopic biopsy specimens of gastric adenocarcinoma were obtained and similarly fixed in buffered formalin. In the case of gastric adenocarcinoma, 4 of 12 specimens were well or moderately differentiated (all intestinal type) adenocarcinoma, whereas 8 of 12 were poorly differentiated (all diffuse type) adenocarcinoma. Specimens of nonneoplastic gastric mucosa distant from the cancer were also obtained for comparison with the neoplastic tissue.

Finally, eight biopsies of Barrett's epithelium from patients who also had esophageal adenocarcinoma were obtained and fixed in Bouin's solution and were found to have high grade dysplasia. These tissues were evaluated as a separate group.

Lectin labeling. 4- $\mu \mathrm{m}$ paraffin-embedded sections were used for these studies. Each section of tissue was deparaffinized in xylene and rehydrated through serial alcohol solutions to PBS. The tissues were labeled with FITC-conjugated lectin in a concentration of $100 \mu \mathrm{g} / \mathrm{ml}$. $50 \mu \mathrm{l}$ of lectin was applied to the tissue section, incubated for $20 \mathrm{~min}$, and washed off with PBS. The tissue sections were then mounted in gelvatol (Montsanto Co., St. Louis, MO) and the slides were examined with a Zeiss epifluorescence microscope.

The lectins used and their corresponding hapten sugars are listed in Table I. The specificity of binding was documented by specific inhibition in the presence of $200 \mathrm{mM}$ hapten sugar. The lectins were obtained commercially from Vector Laboratories (Burlingame, CA) and E-Y Laboratories (San Mateo, CA).

Each of the lectins was applied to each of the 123 specimens collected. Because of the obvious morphological configuration of each specimen, it was not possible to blind the fluorescence microscopist to the identity of the tissues. However, the fluorescence microscopist was blinded to the clinical information regarding each case, and the histopathologist was blinded to the lectin-binding findings while assigning the tissues to the various pathological categories. Prior investigation using this technique has demonstrated a high degree of intra- and interobserver reproducibility $(23,24)$.

The binding of fluorescent lectins to tissues was scored from 0 to $3+$. Zero indicated negative staining, and the most intense degree of labeling was assigned a score of $3+$. Scores of $1+$ and $2+$ referred to weak and intermediate levels of binding, respectively. The fluorescence microscopist identified distinct components of the mucosa within each biopsy and scored them independently. One microscopist scored all of the specimens (Dr. Shimamoto). Schematic representations indicate the components that were identified and scored individually. This method permitted the individual scoring of glycoconjugates that were intracellular, localized within a goblet cell theca, or secreted. It was not possible to clearly distinguish whether the labeling that occurred along the luminal surface of an epithelial cell represented cell membrane labeling or the presence of secreted glycoconjugates (e.g., mucus) that had become adherent to the luminal surface of the cell. Labeling in this region was referred to as membrane-associated. This labeling was recorded independently of secreted mucus that was clearly distinguishable from the membrane-associated labeling.

Glycoconjugate expression was recorded in terms of region within the upper gastrointestinal tract, pathological diagnosis, lectin used, cell population within the biopsy, and intensity of labeling.

Table I. Lectins and Carbohydrate Binding Determinants

\begin{tabular}{ll}
\hline Lectin & Carbohydrate binding determinants \\
\hline PNA & Gal $\beta-1,3$ GalNAc (T antigen) \\
DBA & $\alpha$-GalNAc (blood group A) \\
UEA $_{1}$ & $\alpha$-Fucose (blood group O) \\
s-WGA & GlcNAc \\
LFA & Sialic acid
\end{tabular}

Gal, Galactose; GalNAc, $n$-acetylgalactosamine. 

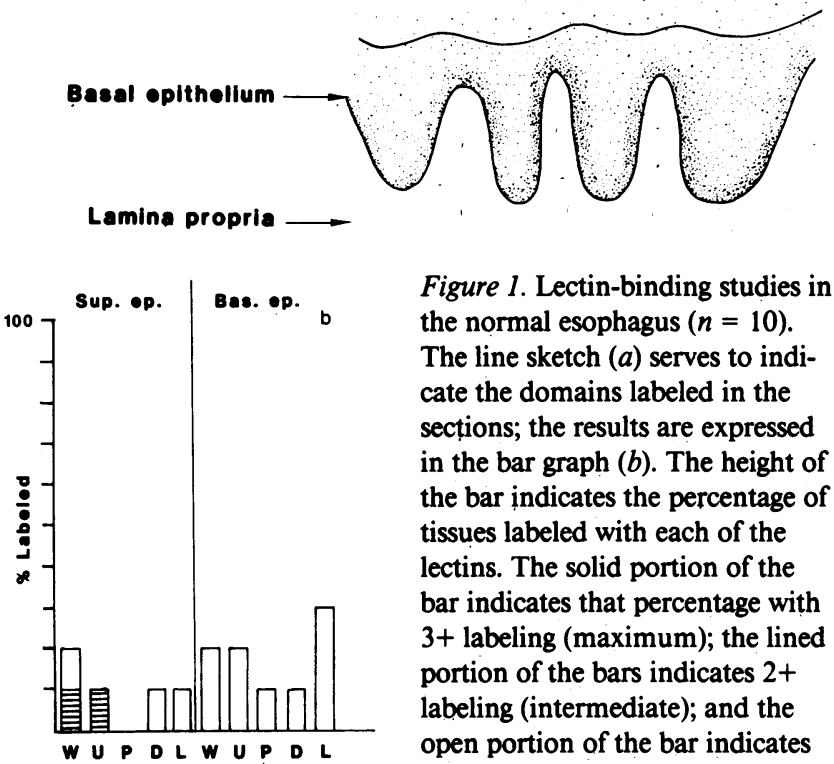

Figure 1. Lectin-binding studies in the normal esophagus $(n=10)$. The line sketch $(a)$ serves to indicate the domains labeled in the sections; the results are expressed in the bar graph $(b)$. The height of the bar indicates the percentage of tissues labeled with each of the lectins. The solid portion of the bar indicates that percentage with $3+$ labeling (maximum); the lined portion of the bars indicates $2+$ labeling (intermediate); and the open portion of the bar indicates $1+$ labeling (weakly present). The

height of the bar represents the percent $(0-100)$ of those tissues labeled. The data are expressed individually for each domain in the tissue section. Sup. ep., Superficial epithelium, i.e., the superficial half of the epithelial layer; Bas. ep., basal epithelium, i.e. the lower half of the epithelial layer. The individual lectins are indicated along the bottom of the graph: W, s-WGA; U, UEA ; P, PNA; D, DBA; and L, LFA (for all figures).

\section{Results}

Esophagus. Fig. $1 a$ is a schematic representation of squamous esophageal mucosa. The superficial and basal portions of the epithelium have been scored separately. Fig. $1 b$ is a bar graph in which the data are recorded in terms of the percentage of the biopsies labeled with each of the lectins in each mucosal zone. The height of the bar indicates the percentage of biopsies labeled by each of the lectins. In the case of esophageal mucosa, $80-90 \%$ of the biopsies showed no labeling with the panel of lectins, most of the labeling was weak, and there were no important differences in labeling between the superficial and basal portions of the epithelium.

Proximal stomach: fundic gland mucosa. Fig. $2 a$ is a schematic representation of the fundic gland mucosa, in which various portions of the mucosa have been independently evaluated. Fig. $2 b$ is a bar graph in which the data are recorded in terms of percentage of the biopsies labeled as discussed above.

It is apparent that succinylated wheat germ agglutinin (sWGA $)^{2}$ (W in the figures), ulex europeus agglutinin ${ }_{1}\left(\mathrm{UEA}_{1}\right)$ ( $U$ in the figures), and peanut agglutinin (PNA) ( $P$ in the figures) labeled the apical cell membrane, the adherent and secreted mucus, the surface epithelium, the pit epithelium, and the parietal cell zone in the upper half of the gland in the

2. Abbreviations used in this paper: $\mathrm{DBA}$, dolichos biflorus agglutinin; GlcNAc, $n$-acetylglucosamine; LFA, limax flavus agglutinin; PNA, peanut agglutinin; s-WGA, succinylated wheat germ agglutinin; UEA $_{1}$, ulex europeus agglutinin $n_{1}$.
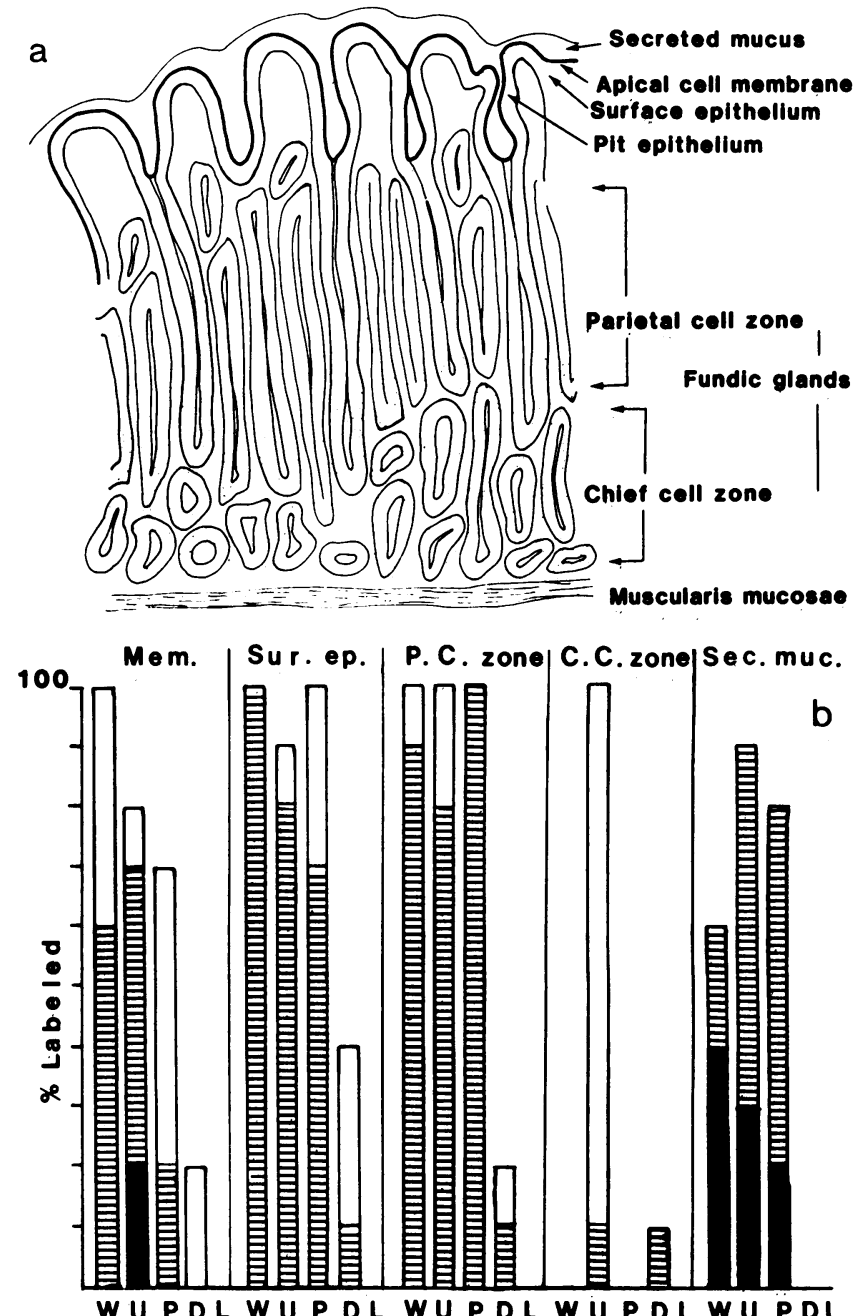

Figure 2. Lectin-binding studies in the normal fundic gland mucosa $(n=10)$. The line sketch $(a)$ serves to indicate the domains labeled in the sections, and the results are expressed in the bar graph $(b)$. The bars and axes are as described in Fig. 1. Mem., Membrane-associated labeling, i.e., that on the cell membrane adjacent to the lumen or secreted mucus adherent to the luminal surface; Sur. ep., labeling in the cytoplasm of the surface epithelium and pit epithelium; P.C. zone, labeling of the parietal cell zone, which is in the upper half of the deep fundic gland; C.C. zone, labeling of the chief cell zone, which is in the lower portion of the fundic gland mucosa; Sec. muc., labeling of secreted mucus. The individual lectins are indicated along the bottom of the graph as described in Fig. 1.

gastric fundus. Dolichos biflorus agglutinin (DBA) (D in the figures) and limax flavus agglutinin (LFA) ( $L$ in the figures) did not tend to label these regions. UEA $A_{1}$, but not s-WGA or PNA, labeled the chief cell zone in the lower part of the fundic gland, which indicated differential expression of carbohydrate structures in this portion of the mucosa. $\mathrm{UEA}_{1}$ labeled all of the epithelium, including the surface, pit, and glandular domains, which indicated the expression of terminal fucosyl residues in all cell types.

Gastric antrum. Labeling in the antrum was generally similar to that in the fundic gland mucosa, so the data have not been independently expressed graphically. s-WGA, UEA $A_{1}$, and PNA prominently labeled the cell membrane, adherent mucus, superficial epithelium, and pit epithelium. These three 
lectins labeled the full length of the antral glands. DBA showed labeling throughout the antral glands, but was $>2+$ in only $20 \%$ of the biopsies. LFA did not bind to any glycoconjugates in this region. Thus, the gastric fundic and antral gland mucosae had a similar expression of carbohydrate structures, with the exception that binding sites for s-WGA and PNA were not seen in the chief cell zones of fundic glands.

Gastric-type columnar metaplasia. Fig. $3 a$ is a schematic representation of regions evaluated in gastric-type columnar metaplasia in the esophagus. Fig. $3 b$ is a bar graph of the lectin-binding results in biopsies with exclusively gastric-type surface epithelium, or with major zones of it in the biopsies. The labeling patterns using s-WGA, UEA $A_{1}$, PNA, DBA, and LFA closely resembled those of the gastric antrum, with the exception of secreted mucus, which showed binding by $\mathrm{s}$-WGA and $\mathrm{UEA}_{1}$ in all cases.

Duodenum. Fig. $4 a$ is a schematic representation of the duodenal epithelium. Fig. $4 b$ is a bar graph of lectin labeling in this region. Although s-WGA, UEA $A_{1}$, and PNA labeled the glycoconjugates adherent to the microvillar membranes of duodenal epithelium, there was a conspicuous absence of binding with these three lectins in the villus and crypt enterocytes of this region. However, s-WGA and UEA $_{1}$ bound to the goblet cell mucin in the villi and crypt of the duodenum. The mucin in the Brunner's glands of this region contrasted sharply with glycoconjugates found elsewhere in the duodenum with intense binding by s-WGA, UEA ${ }_{1}$, and PNA. Binding by DBA and LFA was not seen in this epithelium. Thus, there was a more restricted expression of the carbohydrate structures that bind these lectins in the duodenum, and the glycoconjugates
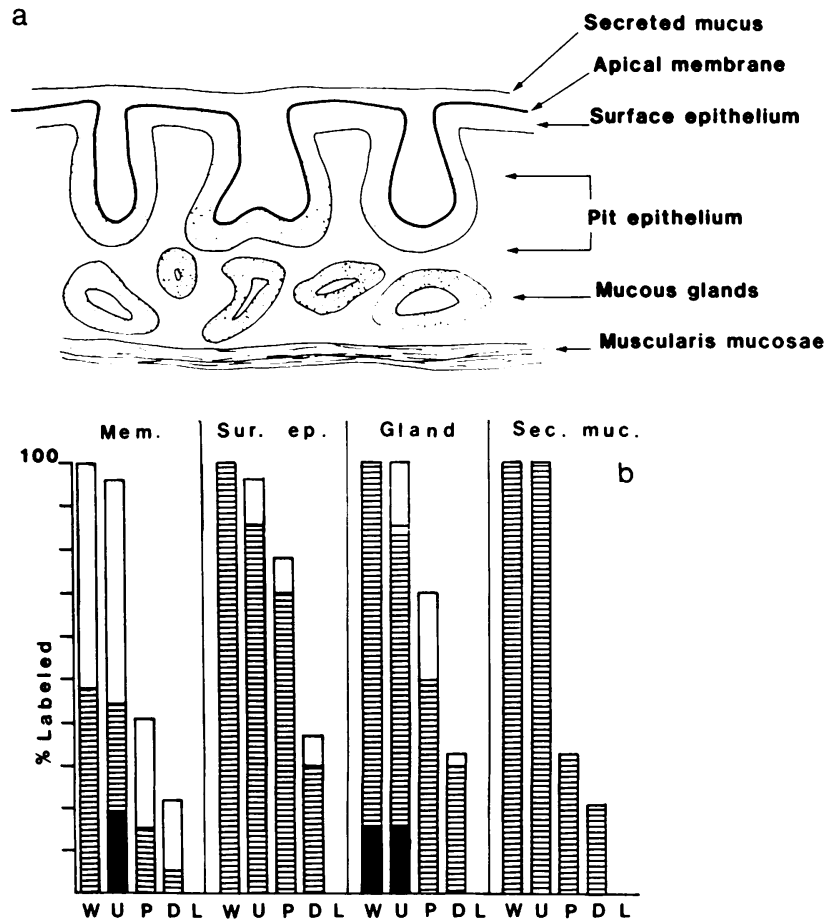

Figure 3. Lectin labeling of epithelial glycoconjugates in gastric-type Barrett's epithelium ( $n=27$ specimens). Mem., Sur. ep., and Sec. muc. refer to lectin labeling in epithelial domains as defined in Fig. 2. Gland, labeling in the underlying (deep) mucous glands. The bars and axes are as defined in Fig. 1.
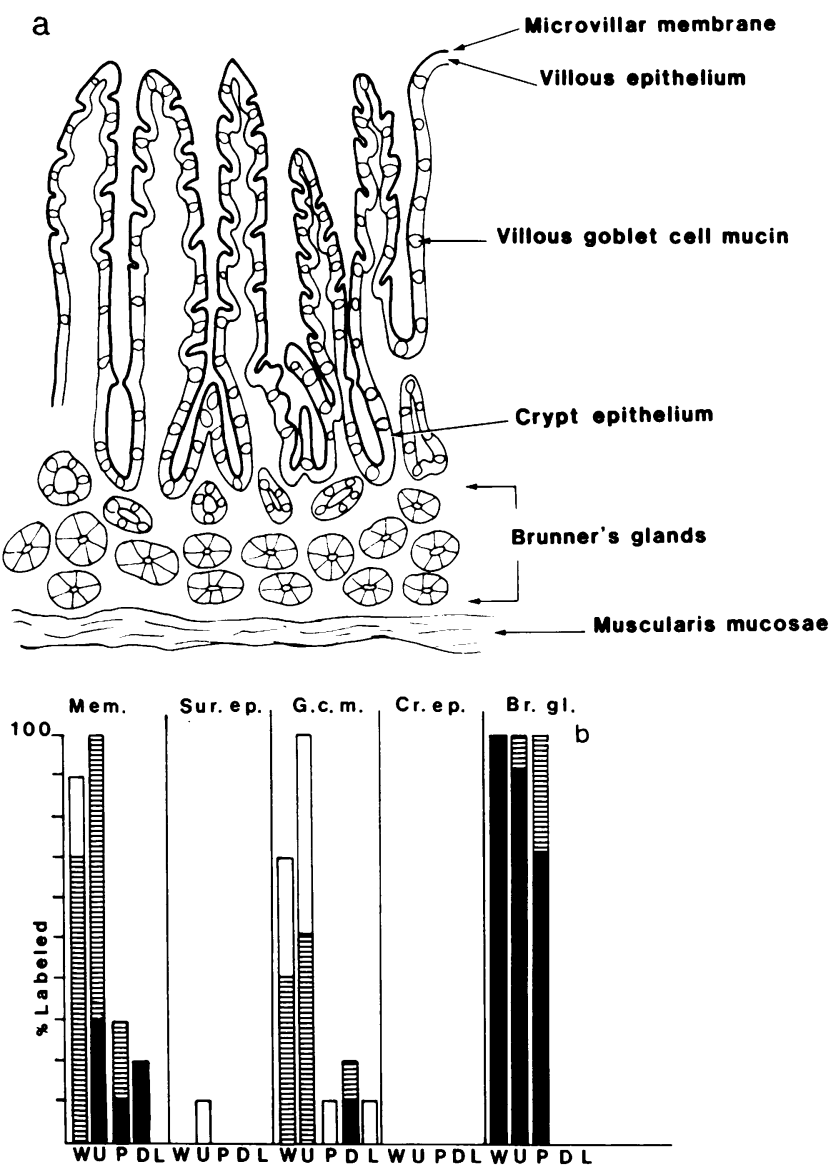

Figure 4. Lectin labeling of epithelial glycoconjugates in the duodenum $(n=10)$. Mem. refers to labeling of the same domains as defined in Fig. 2. Sur. ep. includes that of the villi. G.C.M., Villous and crypt goblet cell mucins; $\mathrm{Cr}$. ep., crypt epithelium; Br. gl., Brunner's glands. The bars and axes are as described in Fig. 1.

found in Brunner's glands contrasted sharply with those synthesized in the overlying epithelium.

Specialized columnar metaplasia. Fig. $5 a$ is a schematic representation of specialized-type columnar metaplasia in the esophagus. Fig. $5 b$ is a bar graph of the lectin-binding studies in this region. The surface, pit, and villous epithelium all shared a uniform pattern of lectin binding in this tissue, and are recorded as surface epithelium in Fig. 5 b. Compared with the findings in nonspecialized columnar metaplasia, this epithelium showed a reduction in binding with the lectins PNA and DBA.

Two differences were seen between glycoconjugates in specialized columnar epithelial metaplasia and those in normal duodenum. First, throughout the epithelium of specialized metaplasia, all of the tissues showed binding by $\mathrm{UEA}_{1}$ and $60 \%$ bound s-WGA, whereas none of these lectins labeled the surface or crypt epithelium in the normal duodenum. Secondly, in approximately half of the metaplastic tissues, the binding of LFA to goblet cell mucin was seen, a feature present in none of the normal tissues.

Dysplasia in Barrett's esophagus. Five biopsies of Barrett's esophagus not associated with esophageal cancer contained foci of cytological abnormalities that fell into the poorly defined category of "low grade dysplasia." Four of these were 

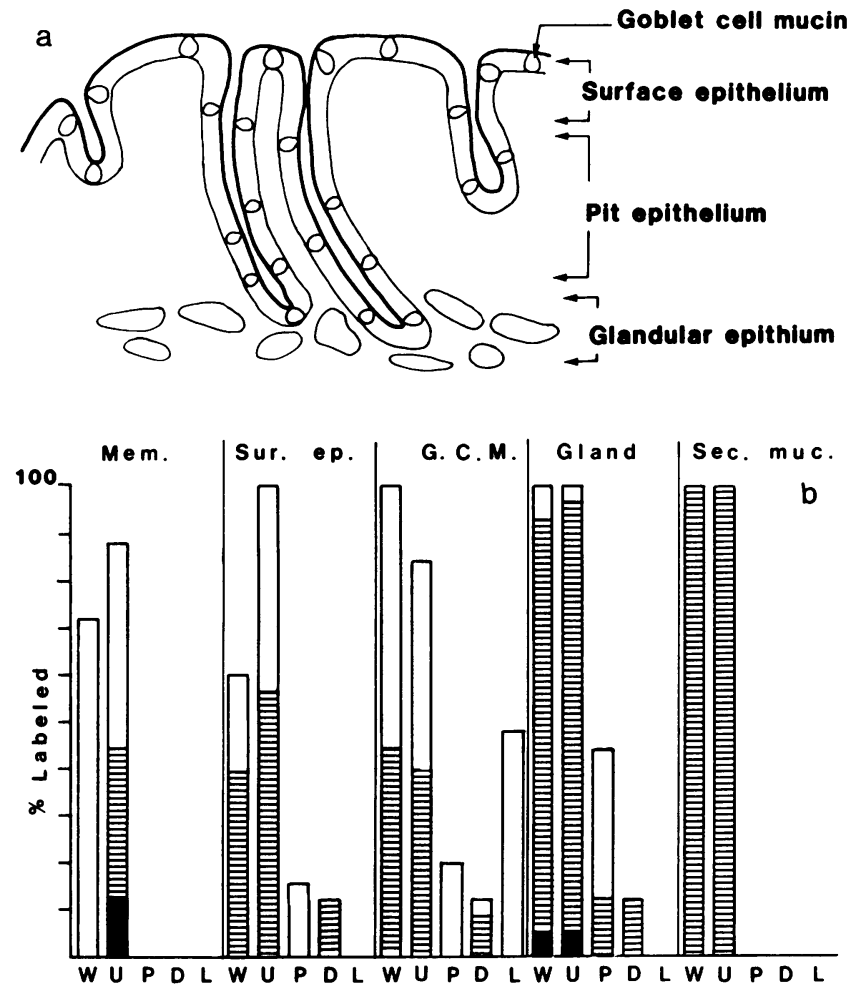

Figure 5. Lectin labeling of epithelial glycoconjugates in specialized Barrett's epithelium ( $n=25$ specimens). Mem. and Sec. muc. refer to epithelial domains as defined in Fig. 1. Sur. ep. in this figure refers to labeling in the surface, pit, and villous-type epithelium. G.C.M., Labeling of goblet cell mucin; Gland, labeling of glycoconjugates in the underlying mucous glands. The bars and axes are as described in Fig. 1.

seen in gastric-type columnar metaplasia and one was in specialized-type metaplasia. When analyzed separately, these tissues had the same lectin labeling patterns as were seen in the other gastric-type and specialized-type metaplastic tissues without foci of low grade dysplasia, including labeling with the lectin LFA. Because of the small numbers and difficulty in making this diagnosis, these biopsies were not considered further as separate groups.

Fig. 6 depicts the lectin labeling of glycoconjugates in high grade dysplasia in Barrett's epithelium from patients with adenocarcinoma of the esophagus. In these tissues, there was a complete loss of the expression of lectin-binding glycoconjugates in the superficial epithelium and the upper half of the glandular elements. Note the "normal" expression of binding sites for s-WGA, UEA 1 , PNA, and DBA in the deep mucous glands of this epithelium. The lectin LFA did not bind to any tissue in this group.

Esophageal adenocarcinoma. The labeling of the surface membrane or cytoplasmic glycoconjugates among the eleven moderately to well differentiated esophageal adenocarcinomas is depicted in Fig. 7. Again, labeling by s-WGA was seen in all the tumors, whereas there were intermediate degrees of labeling with $\mathrm{UEA}_{1}$ and PNA. Weak labeling by DBA was seen in only one tumor and no tumor bound LFA. Among the adenocarcinomas of the esophagus, most of the labeling occurred on the cell membrane that made up the lumenal perimeter of the malignant gland. Cytoplasmic labeling was much less promi-

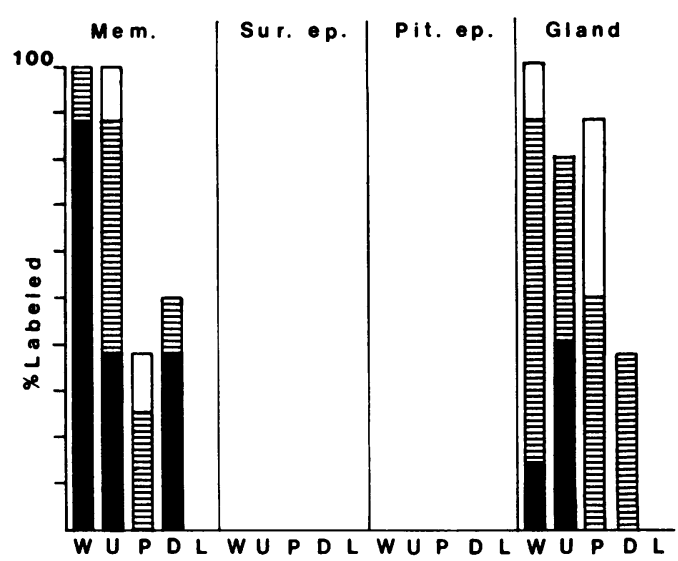

Figure 6. High grade dysplasia in Barrett's epithelium ( $n=8$ specimens). Mem. and Sur. ep. in this bar graph refer to epithelial labeling domains as defined in Fig. 2. Pit. ep. refers to labeling in the epithelium of the pits or crypts. Gland refers to labeling in deep mucous glands. The bars and axes are as described in Fig. 1.

nent than in the gastric carcinomas, and labeling of secreted mucins was not seen.

For both benign and malignant tissues, there did not appear to be any differences in labeling related to the fixative, i.e., Bouin's or formalin.

Gastric adenocarcinoma. The labeling of cytoplasmic, cell surface membrane, and secreted glycoconjugates in moderately and well differentiated gastric adenocarcinomas is indicated in the left panel of Fig. 8. In these four cancers, the binding of s-WGA, UEA ${ }_{1}$, and PNA to cytoplasmic, membrane-associated, and secreted glycoconjugates was prominent, whereas labeling by DBA and LFA was not seen. The right panel of Fig. 8 depicts lectin labeling in the eight poorly differentiated gastric adenocarcinomas. In this instance, s-WGA and PNA prominently labeled both the cell surface membrane and cytoplasm. In these poorly differentiated adenocarcinomas, no well-formed glands or secreted mucins were visualized. The labeling by $\mathrm{UEA}_{1}$ was absent among all the poorly differentiated tumors, which contrasted with the labeling seen in the moderately and well-differentiated gastric adenocarcinomas.

Nonneoplastic gastric mucosa was examined in eight specimens from four of the resected specimens of gastric cancer, and was found to have the same glycoconjugate expression as those gastric specimens from patients with Barrett's esophagus.

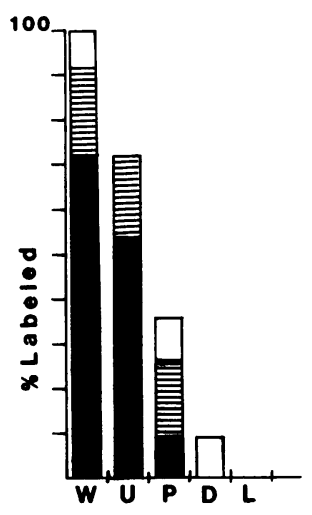

Figure 7. Labeling of cell surface membrane and cytoplasmic glycoconjugates in adenocarcinoma of the esophagus ( $n$ $=11$ specimens). The bars and axes are as described in Fig. 1. 

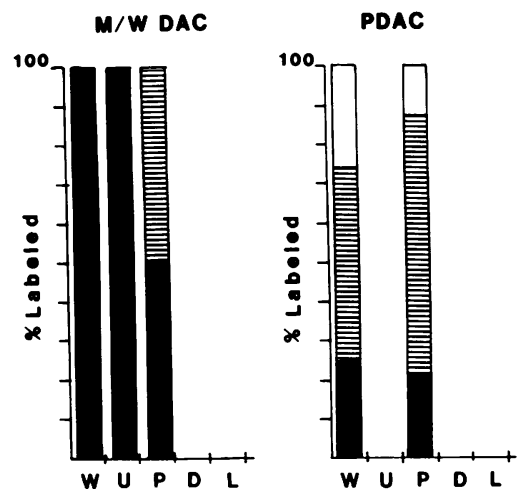

Figure 8. Labeling of cytoplasmic, cell surface membrane, and secreted glycoconjugates in gastric adenocarcinoma $(n=12$ specimens). Labeling of glycoconjugates in moderately and welldifferentiated gastric adenocarcinoma $(M / W$ DAC) is depicted on the left bar graph $(n=4$ specimens). The bars and axes are as described in Fig. 1. Labeling of glycoconjugates in poorly differentiated adenocarcinoma (PDAC) is depicted in the right bar graph ( $n=8$ specimens). Secreted glycoconjugates were not labeled in any of these specimens. The bars and axes are as described in Fig. 1.

Metaplasia in the stomach and duodenum. Foci of intestinal metaplasia were encountered in three gastric biopsies. In all three there was an abrupt change in glycoconjugate expression from that typical of the stomach to that characteristic of duodenum. Fig. 9 is a photomicrograph that illustrates the parallel changes in morphology and glycoconjugate expression.

Similarly, one focus of gastric metaplasia was found in an otherwise normal specimen of duodenal mucosa. The binding of lectins to the metaplastic tissue underwent an abrupt change, and resembled that seen in gastric epithelium.

\section{Discussion}

The results indicate that the esophagus, stomach, and duodenum all have their characteristic "fingerprint" with respect to terminal glycosylation patterns as measured by the binding of lectins. Metaplastic tissues synthesize glycoconjugates true to the morphological configuration of the tissue, regardless of its anatomic location in the upper gastrointestinal tract. The development of low grade dysplasia is not accompanied by important changes in glycosylation, however high grade dysplasia and carcinoma have their own characteristic patterns of glycosylation, including a variable deletion of several terminal carbohydrate structures.

The normal stomach has prominent expression of structures that bind s-WGA (which recognizes $n$-acetylglucosamine [GlcNAc] residues), $\mathrm{UEA}_{1}$ (which recognizes the alpha-linked fucose, such as that in the blood group $\mathrm{O}$ or $\mathrm{H}$ structure), and PNA (which recognizes the exposed disaccharide galactose beta 1,3n-acetylgalactosamine, or the T-antigen). Structures recognized by DBA (which binds the terminal alpha-linked $n$-acetylgalactosamine in blood group $A_{1}$ ) are more selectively expressed, and those binding LFA (sialic acid) are not seen. A review of the patients' blood types revealed that DBA labeled only biopsies from blood group A patients (data not presented). Although this is not surprising, it differs from the colon, in which DBA binds to colonic mucins from patients with all $\mathrm{ABO}$ blood types $(8,9)$. The labeling by LFA was potentially more interesting, as it was only found in specialized columnar metaplasias, and not in any normal tissues. However, LFA did not bind to high grade dysplasia or cancer, which limited its value as an indicator of imminent neoplasia. A second sialic acid-binding lectin, that from Limulus polyphemus, was used on each of the tissues in this study, but bound to none of them (data not presented).

This work provides corroborating evidence for the structures characterized by other biochemical techniques in which several heterogeneous $\mathrm{H}+$, Lewis $^{\mathrm{b}}+$ blood group antigens have been detected (25), and those in which blood group A structures have been isolated (26) from purified human gastric mucins. Lewis antigens have also been localized immunohistochemically in the stomach and duodenum (27). Additionally, a variety of shorter oligosaccharide structures terminating in GlcNAc have been characterized by nuclear magnetic resonance spectroscopy in canine gastric mucins (28) that may represent partially glycosylated precursor chains, and may account for at least some of the heterogeneity of gastrointestinal mucins. The work presented here provides detail regarding the precise localization of the expression of different forms of mucin-type glycoconjugates, and adds suggestive evidence on the relative quantitative expression of different oligosaccharide species in different mucosal "microenvironments."

In spite of the morphological differences between the fundic and antral gland mucosae, only minor differences in glycoconjugate expression were seen, specifically the absence of binding sites for PNA and paucity of those for s-WGA in the chief cell zone in the lower portion of the fundic glands. This latter observation suggests a selective expression of glycoconjugates by chief cells. No change in the pattern of glycoconjugate expression was seen in the mucous neck cell region, which is thought to be the proliferative region of gastric epithelium (29). The expression of different glycoconjugate structures throughout the crypt unit has been reported in the small intestine $(1,5)$ and colon $(7,9)$, which may reflect either differentiation-dependent or other functionally dependent changes in glycosylation.

The duodenal mucosa differed from gastric mucosa with a marked reduction in lectin-binding sites in both the villus and crypt regions. Brunner's glands differed strikingly from the overlying epithelium in that glycoconjugates in the former were bound by s-WGA, UEA, and PNA.

In metaplastic epithelium, differentiated tissue is found in an inappropriate location. These lectin-binding studies have allowed a comparison between glycoconjugate structures in metaplasia and those found in normal tissues. Glycoconjugates in columnar epithelial metaplasia demonstrated several interesting similarities and differences when compared with the corresponding normal tissues. Gastric-type Barrett's epithelium showed the same labeling pattern as was seen in normal gastric mucosa. This is not especially surprising since most of the biopsies with gastric-type epithelium were from the lower esophageal region or within $2 \mathrm{~cm}$ of it. This may not reflect metaplasia in all instances; it may reflect an imperfect or discrepant (squamo-columnar) histologic junction in relation to the location of the lower esophageal sphincter region.

The specialized type of epithelium differed from normal duodenal mucosa in that increased $\mathrm{UEA}_{1}$ and s-WGA binding was seen throughout the metaplastic tissue compared with surface and crypt epithelium in the normal duodenum. In 


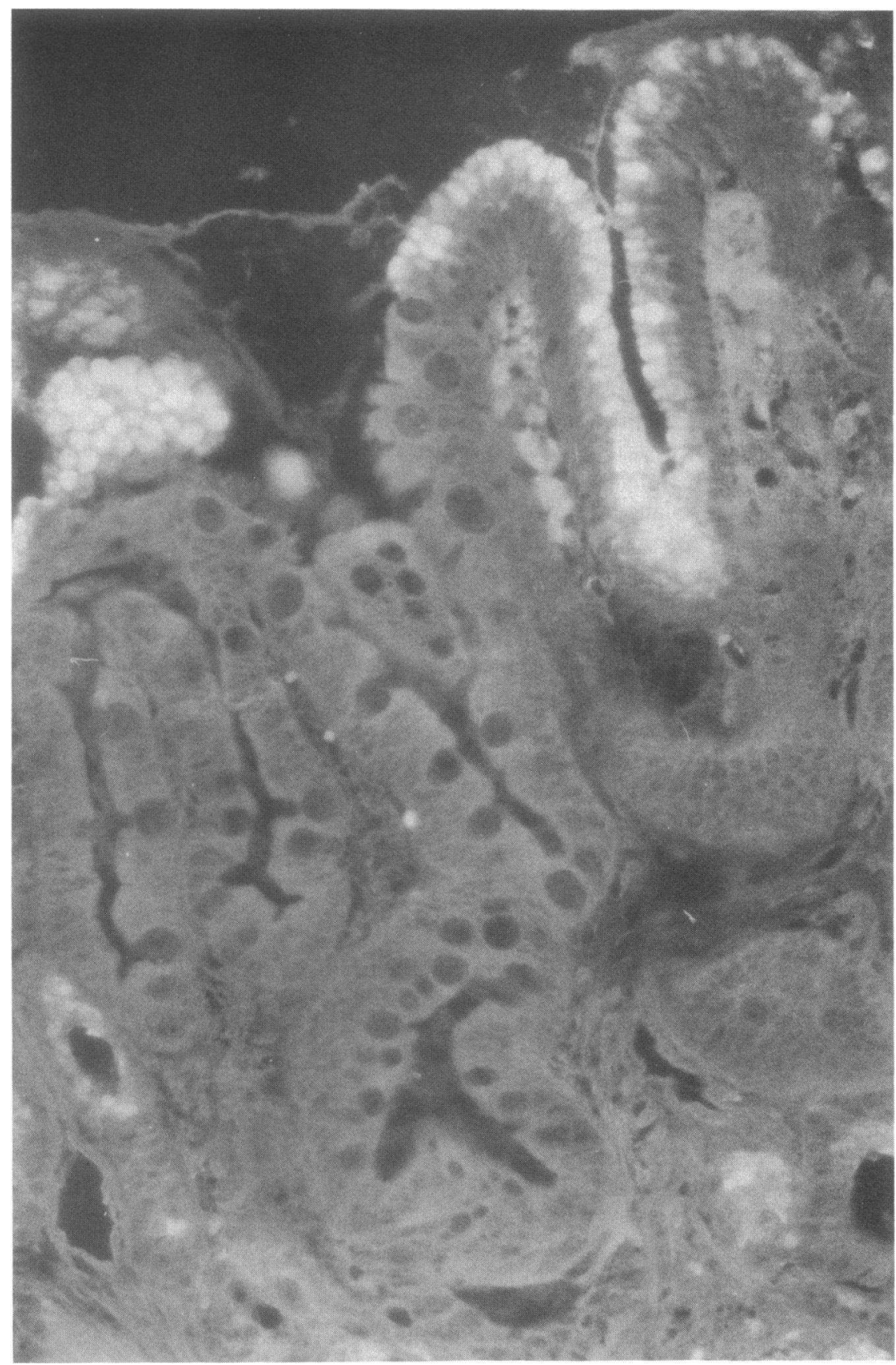

Figure 9. Fluorescent photomicrograph of tissue section labeled with fluorescent lectin $(\times 300)$. Antral gland biopsy stained with fluorescent PNA demonstrates normal surface epithelium in the upper right and a focus of intestinal metaplasia on the left half of the figure. Note that PNA labels the normal epithelium on the right, but labels neither the surface epithelium nor goblet cell mucin in the intestinalized portion on the left. This abrupt change in labeling is characteristic of that observed among the various foci of metaplasia in the biopsies. general the glycoconjugate structures expressed in metaplastic tissue more closely resembled those of normal rather than neoplastic tissues.

Dysplasia is a morphological term which refers to tissue that is thought to have a defect in differentiation, and it is perhaps the earliest identifiable form of neoplasia. The presence of low grade dysplasia had no important effects on glycoconjugate expression in this study. This may reflect the diffculty in distinguishing low grade dysplasia from nondysplastic columnar metaplasia (i.e., ordinary Barrett's mucosa) (30). However, a distinct change was seen in high grade dysplasia, in which the expression of several terminal carbohydrate structures, those bound by s-WGA, UEA 1 , PNA, and DBA, were not expressed in the surface epithelium. This was of particular interest because these carbohydrate structures were expressed in normal gastric epithelium, whereas binding sites for DBA and $\mathrm{UEA}_{1}$ tended not to be expressed in the glycoconjugates of adenocarcinoma of the esophagus and stomach. This could be accounted for by a failure of terminal glycosylation in high grade dysplasia and carcinoma. However, the synthesis of a more complex carbohydrate structure not recognized by the lectins used in this study could produce similar results. In any event, the perturbation in terminal glycosylation patterns is first seen in high grade, rather than low grade, dysplasia. Detailed structural studies on glycoconjugates from these types of tissues will be necessary to unravel the mechanisms that underlie the change in glycoconjugate structure seen in neoplastic tissues. 
It was of additional interest that $\mathrm{UEA}_{1}$ binding sites were consistently expressed in well-differentiated gastric adenocarcinoma but not in poorly differentiated gastric adenocarcinoma. DBA binding was not seen in either morphological variant of gastric cancer, which is consistent with previous reports of a deletion of blood group A activity in gastric carcinoma (31). Poorly differentiated adenocarcinoma was characterized by a partial reduction of s-WGA and PNA binding sites as well. In fact, the progression from normal to fully malignant tissue was generally characterized by a stepwise deletion of terminal glycosylation structures in mucin-type glycoconjugates, an observation consistent with other recent reports $(32,33)$.

In summary, a glycoconjugate profile has been defined for each distinct epithelial cell population in the stomach, duodenum, and columnar-lined esophagus (Barrett's esophagus). Glycoconjugate expression in epithelial metaplasia was found to resemble the structures expressed in normal tissues. Minor alterations in glycoconjugate expression were found in low grade dysplasia of the columnar-lined esophagus, however a restriction in the expression of glycoconjugates was seen in high grade dysplasia. Restricted glycoconjugate expression was also found in adenocarcinomas of the esophagus and stomach. These studies demonstrate that glycosylation of mucin-type glycoconjugates is preserved in epithelial metaplasia, but is aberrant in the settings of high grade dysplasia and cancer. Fluorescent lectin studies on tissue sections permit the detailed localization of glycoconjugates structures within different cell populations in normal, metaplastic, and neoplastic tissues. This experimental approach provides information on epithelial microdomains not generally available with techniques directed towards more complete biochemical characterization.

\section{Acknowledgments}

The authors are indebted to Ms. Brooks Comstock for her patient manuscript preparation, to Myriam Marin for the histological preparations, to William O. Dobbins III, M.D., and the Histochemistry Core at the Ann Arbor VA Medical Center for histological preparations; to Darlene Scott for help in retrieving many tissue blocks and sections, and to Barbara Dyko, Ph.D. for the mucosal line sketches and graphs.

This work was supported in part by the Medical Research Service of the Veterans Administration, by U.S. Public Health Service grant CA 39233, awarded by the National Cancer Institute, Department of Health and Human Services, by U.S. Public Health Service grant P30 AM 34933, and by National Institutes of Health grant DK-17328 to the Center for Ulcer Research and Education.

\section{References}

1. Isselbacher, K. J. 1974. The intestinal cell surface: some properties of normal, undifferentiated and malignant cells. Ann. Intern. Med. $81: 681-686$

2. Carlson, D. M., R. N. Iyer, and J. Mayo. 1970. Carbohydrate compositions of epithelial mucins. In Blood and Tissue Antigens. D. Aminoff, editor. Academic Press, Inc., New York. 229-247.

3. Podolsky, D. K. 1985. Oligosaccharide structures of human colonic mucin. J. Biol. Chem. 260:8262-8271.

4. Podolsky, D. K. 1985. Oligosaccharide structures of isolated human colonic mucin species. J. Biol. Chem. 260:15510-15515.

5. Weiser, M. M. 1973. Intestinal epithelial cell surface membrane glycoprotein synthesis. I. An indicator of cellular differentiation. $J$. Biol. Chem. 248:2536-2541.

6. Rampal, P., J. T. LaMont, and J. S. Trier. 1978. Differentiation of glycoprotein synthesis in fetal rat colon. Am. J. Physiol. 235(Suppl. 2):E207-E212.

7. Freeman, H. J., R. Lotan, and Y. S. Kim. 1980. Application of lectins for detection of goblet cell glycoconjugate differences in proximal and distal colon of the rat. Lab. Invest. 42:405-412.

8. Bresalier, R. S., C. R. Boland, and Y. S. Kim. 1985. Regional differences in normal and cancer-associated glycoconjugates of the human colon. J. Natl. Cancer Inst. 75:249-260.

9. Boland, C. R., C. K. Montgomery, and Y. S. Kim. 1982. Alterations in human colonic mucin occurring with cellular differentiation and malignant transformation. Proc. Natl. Acad. Sci. USA. 79:20512055.

10. Feizi, T., H. C. Gooi, R. A. Childs, J. K. Picard, K. Uemura, L. M. Loomes, S. J. Thorpe, and E. T. Hounsell. 1984. Tumour-associated and differentiation antigens on the carbohydrate moieties of mucin-type glycoproteins. Biochem. Soc. Trans. 12:591-596.

11. Podolsky, D. K., and K. J. Isselbacher. 1983. Composition of human colonic mucin. Selective alteration in inflammatory bowel disease. J. Clin. Invest. 72:142-153.

12. Younan, F., J. Pearson, A. Allen, and C. Venables. 1982. Changes in the structure of the mucous gel on the mucosal surface of the stomach in association with peptic ulcer disease. Gastroenterology. 82:827-831.

13. Smith, R. R. L., S. R. Hamilton, J. K. Boitnott, and E. L. Rogers. 1984. The spectrum of carcinoma arising in Barrett's esophagus. A clinicopathologic study of 26 patients. Am. J. Surg. Pathol. 8:563-573.

14. Paull, A., J. S. Trier, M. D. Dalton, R. C. Camp, P. Loeb, and R. K. Goyal. 1976. The histologic spectrum of Barrett's esophagus. $N$. Engl. J. Med. 295:476-480.

15. Herbst, J. J., M. M. Berenson, D. W. McCloskey, and W. C. Wiser. 1978. Cell proliferation in esophageal columnar epithelium (Barrett's esophagus). Gastroenterology. 75:683-687.

16. Jass, J. R. Mucin histochemistry of the columnar epithelium of the oesophagus: a retrospective study. J. Clin. Pathol. 34:866-870.

17. Peuchmaur, M., F. Potet, and D. Goldfain. 1984. Mucin histochemistry of the columnar epithelium of the oesophagus (Barrett's oesophagus): a prospective biopsy study. J. Clin. Pathol. 37:607-610.

18. Lee, R. G. 1984. Mucins in Barrett's esophagus: a histochemical study. Am. J. Clin. Pathol. 81:500-503.

19. Boland, C. R., C. K. Montgomery, and Y. S. Kim. 1982. A cancer-associated mucin alteration in benign colonic polyps. Gastroenterology. 82:664-72.

20. Boland, C. R., and D. J. Ahnen. 1985. Binding of lectins to goblet cell mucin in malignant and premalignant colonic epithelium in the CF-1 mouse. Gastroenterology. 89:127-137.

21. Weinstein, W. M., and T. Hill. 1985. Gastrointestinal mucosal biopsy. In Bockus Textbook of Gastroenterology. 4th ed. J. Berk, editor. W. B. Saunders Co., Philadelphia. 626-644.

22. Hassall, E., W. M. Weinstein, and M. E. Ament. 1985. Barrett's esophagus in childhood. Gastroenterology. 89:1331-1337.

23. Boland, C. R., P. Lance, B. Levin, R. H. Riddell, and Y. S. Kim. 1984. Abnormal goblet cell glycoconjugates in rectal biopsies associated with a increased risk of neoplasia in patients with ulcerative colitis. Early results of a prospective study. Gut. 25:1364-1371.

24. Boland, C. R., and P. Lance. 1984. Abnormal goblet cell mucin in nonneoplastic colonic disease. Clin. Res. 32:282A. (Abstr.)

25. Slomiany, B. L., E. Zdebska, and A. Slomiany. 1984. Structural characterization of neutral oligosaccharides of human $\mathrm{H}^{+} \mathrm{Le}^{\mathrm{b}+}$ gastric mucin. J. Biol. Chem. 259:2863-2869.

26. Slomiany, A., E. Zdebska, and B. L. Slomiany. 1984. Structures of the neutral oligosaccharides isolated from A-active human gastric mucin. J. Biol. Chem. 259:14743-14749.

27. Lemieux, R. U., D. Baker, W. M. Weinstein, and C. M. Switzer. 
1981. Artificial antigens. Antibody preparations for the localization of Lewis determinants in tissues. Biochemistry. 20:199-205.

28. Van Halbeek, H., L. Dorland, J. F. G. Vliegenthart, N. K. Kochetkov, N. P. Arbatsky, and V. A. Derevitskaya. 1983. Characterization of the primary structure and the microheterogeneity of the carbohydrate chains of porcine blood-group $\mathrm{H}$ substance by $500-\mathrm{MHz}$ ${ }^{1}$ MR spectroscopy. Eur. J. Biochem. 127:21-29.

29. Lipkin, M. 1981. Proliferation and differentiation of gastrointestinal cells in normal and disease states. In Physiology of the Gastrointestinal Tract. L. R. Johnson, editor. Raven Press, New York. 145-168.

30. Reid, B. J., R. C. Haggitt, C. E. Rubin, G. Roth, C. M. Surawicz, G. VanBelle, K. Lewin, W. M. Weinstein, D. A. Antonioli, H. Goldman, W. MacDonald, and D. Owen. 1985. Criteria for dysplasia in Barrett's esophagus: a consensus study. Gastroenterology. 88:1552. (Abstr.)

31. Finan, P. J., D. G. D. Wight, E. S. Lennox, S. H. Sacks, and N. M. Bleehen. 1983. Human blood group isoantigen expression on normal and malignant gastric epithelium studied with anti-A and anti-B monoclonal antibodies. J. Natl. Cancer Inst. 70:679-685.

32. Fischer, J., G. Uhlenbruck, P. J. Klein, M. Vierbuchen, and R. Fischer. 1984. Characterization of glycoconjugates of human gastrointestinal mucosa by lectins. II. Lectin binding to the isolated glycoproteins of normal and malignant gastric mucosa. J. Histochem. Cytochem. 32:690-696.

33. Bur, M., and W. A. Franklin. 1985. Lectin binding to human gastric adenocarcinomas and adjacent tissues. Am. J. Pathol. 119:279-287. 